\section{GP21 UNDIFFERENTIATED NASOPHARYNGEAL CARCINOMA IN CHILDREN AND YOUNG ADULTS: EXPERIENCE IN THE RADIOTHERAPY DEPARTMENT OF THE FES UNIVERSITY HOSPITAL CENTER}

Ghita Chebihi Hassani*, Abdesslam Alami, Zineb Alami. Radiotherapy department in University hospital center of FEZ, FEZ, Morocco

10.1136/archdischild-2019-epa.88

Nasopharyngeal malignant tumors are mainly represented in pediatrics by undifferentiated carcinoma nasopharyngeal tumors (UCNT). It is a rare entity. The aim of our work is to analyze the epidemiological, clinical, therapeutic and evolutionary aspect of children and young $\leq 20$ years with nasopharyngeal cancer.

Patients and methods This is a retrospective study of 38 patients diagnosed with a UCNT between January 2012 and December 2018 and treated in the department of oncologyradiotherapy of the CHU Hassan II of Fez.

Results The age of our patients was between 10 and 19 years, with a middle age 14.7 years, the most affected age group was 10 to 15 years $(70 \%)$, the sex ratio was 1.2 . Diagnosis is often delayed with a revealing symptoms dominated by cervical adenopathy (85\%). $85 \%$ of patients were classified T3-T4 and $60 \%$ were classified N2-N3 with 4 metastatic patients. Treatment was based on neoadjuvant chemotherapy (in $95 \%$ of patients) as well as on concomitant radio- chemotherapy. 3 patients were lost to follow-up. Acute toxicity was characterized by radio mucititis (100\%) and radio dermatitis (60\%) in patients receiving IMRT radiotherapy, hyposialia (55\%), hypoacusis $(44 \%)$ and trismus\%) for those who received 3D conformal radiotherapy, with a lower delayed toxicity for IMRT (only 3 patients who presented with hyposialia). The evolution was marked by a good loco regional control in 55\%, a loco regional recurrence in $10 \%$, and remote metastasis in $15 \%$. Thus, the overall survival was $85 \%$ and the survival without relapse was $50 \%$.

Discussion The prognosis of this cancer remains poor considering the locally advanced forms (high frequency of stages III and IV) and the frequency of recurrences.

Conclusion Improving the prognosis of this cancer requires early diagnosis and more accessible and developed medical infrastructures. The recent advances in conformational radiotherapy by intensity modulation will have to be the main technique of treatment of all our patients.

\section{GP22 LUNG ULTRASOUND IN NEONATES AND BABIES: IS IT AN ACCURATE TOOL FOR IDENTIFICATION OF LUNG DISEASE IN PATIENTS WITH CONGENITAL HEART DEFECTS}

Ivan Akinshin, Elena Sinelnikova*, Ahlam Mohammad, Emilia Stolova, Irina Solodkova, Tatiana Kornishina, Vyacheslav Chasnyk. Saint-Petersburg State Pediatric Medical University, Saint-Petersburg, Russian Federation

\subsection{6/archdischild-2019-epa.89}

Introduction Lung diseases (LDs) are the most common causes of life-threatening respiratory failure in babies, while mortality of it gets up to $35 \%$. Chest X-ray is the most valuable imaging modality for the diagnosis of LDs, but recently lung ultrasound (LUS) has been introduced, and several studies have already confirmed LUS to be an accurate and reliable method for the diagnosis of LD in infants. The goal of this study was to evaluate errors of hyper- and hypodiagnostics of LD using LUS in neonates and babies with primary LD and LD secondary to congenital heart disease (CHD).

Methods We analyzed the results of 241 ultrasonic examinations (heart and lungs) of 131 neonates and babies aged 1 246 days of life. Primary PD (neonatal respiratory distress syndrome - NRDS, bronchopulmonary dysplasia and neonatal pneumonia - NP) had 39 patients, CHD (complete atrioventricular canal ventricular septal defect, atrial septal defect, patent ductus arteriosus, coarctation of the aorta) had 47 patients, diseases without heart and pulmonary involvement (gastrointestinal and urinary tract pathology) had 45 patients. A quarter of patients with CHD also had PDs. LUS was performed according to recommendations of Lichtenstein et al. (2004), Picano et al.(2006). We used standard programs to verify the diagnosis. In order to calculate the errors of hyperand hypodiagnostics of LD regression modeling and classification trees (Statistica for Windows) were used to process the description of lungs and heart in terms of ultrasound examination together with information regarding gender, mode of delivery, gestational age, body weight and length as well as laboratory and instrumental characteristics.

Results Our results confirmed the level of 94-95\% sensitivity and specificity of LUS for identification of RDS and NP, which is almost the same as in thoracic X-ray. Regression models identified $76 \%$ as maximal explained variance for interstitial syndrome described in terms of LUS. LUS explained $42 \%$ of variance of right ventricular dimensions. Error of hyper- and hypodiagnostics of pulmonary hypertension reached $0 \%$ and $27 \%$ respectively, while interstitial syndrome was more informative than alveolar consolidation (score: 22 versus 62 correspondingly). LUS is not useful for differential diagnosis of primary and secondary LD in neonates (errors of hyper- and hypodiagnostics $16 \%$ and $35 \%$ correspondingly) Conclusions Our results confirmed usefulness and feasibility of LUS in diagnostics of NP and NRDS. In addition, LUS is accurate enough for identification of pulmonary hypertension in patients with CHD.

\section{GP23 PERCUTANEOUS CLOSURE OF PATENT DUCTUS ARTERIOSUS: SINGLE CENTER EXPERIENCE}

Birsen Ucar*, Pelin Kosger, Hikmet Kiztanir. Eskisehir Osmangazi University Faculty of Medicine, Department of Pediatric Cardiology, Eskisehir, Turkey

10.1136/archdischild-2019-epa.90

Aim In this study, we aimed to evaluate our clinical experiences in the percutaneous closure of patent ductus arteriosus (PDA) in the pediatric population with our early and late results.

Method A total of 156 patients who underwent percutaneous PDA closure between March 2004 and January 2018 were included in the study. Clinical, echocardiographic and angiographic findings of patients, characteristics of procedures, complications, early and late results were evaluated.

Results The mean age of patients undergoing PDA closure was $4.5 \pm 4$ years (1.5 months-17 years), with an average weight of $17.8 \pm 12.1 \mathrm{~kg}(2.3-68 \mathrm{~kg})$. The PDA type was conical in $117(75 \%)$ patients, tubular in $34(21.7 \%)$, window type in 4 (2.5) patients and complex in $1(0.6 \%)$ patient. In 150 $(96.1 \%)$ patients, the procedure was successful, PDA was 